\title{
Avant-propos
}

Ce livre est issu de cours donnés à l'Université de Nice-Sophia Antipolis dans les années 1970/1980 en maîtrise de physique et en DEUG MP deuxième année, et ensuite dans les années 2000 en licence (L3) et en master de physique M1 et M2. Il est divisé en un tome 1 : "Fondements" et un tome 2 : "Applications". Les dix premiers chapitres du premier tome correspondent à un cours standard de mécanique quantique niveau L3, tandis que le chapitre 11 et le deuxième tome sont plutôt du niveau M1 et M2. Le livre contient environ 200 exercices de longueur et de difficulté variées; plus des trois quarts de ces exercices ont été effectivement utilisés pour des séances de travaux dirigés ou des examens et une sélection de corrigés est disponible à la fin du second tome. En plus des étudiants de licence et de master et ceux des Écoles d'Ingénieurs, ce livre est susceptible d'intéresser un large public de physiciens : étudiants en thèse, chercheurs, enseignants du second degré ou du supérieur qui souhaitent trouver une introduction à la littérature récente ou simplement rafraîchir leurs connaissances en physique quantique.

L'organisation du livre diffère notablement de celle des textes classiques, qui prennent tous comme point de départ l'équation de Schrödinger, ce qui oblige à exposer les principes de base de la mécanique quantique dans un cas qui n'est pas le plus simple et a l'inconvénient de masquer ces principes par des calculs souvent fastidieux. Je me suis efforcé au contraire d'introduire les fondements de la mécanique quantique sur les exemples les plus simples et l'équation de Schrödinger apparaît seulement au chapitre 8. L'approche suivie consiste à mener jusqu'à à son terme la logique qu'avait adoptée Feynman (Feynman et al. [1965]) : développer au maximum un traitement algébrique et exploiter les symétries, en présentant la mécanique quantique dans son cadre autonome, sans faire référence à la physique classique. Cette logique a de nombreux avantages.

- L'approche algébrique permet de traiter des problèmes simples dans des espaces de dimension finie, par exemple de dimension deux : polarisation d'un photon, spin $1 / 2$, atome à deux niveaux... 
- Elle permet d'énoncer de la façon la plus claire les postulats de la mécanique quantique, en séparant ce qui est fondamental de ce qui ne l'est pas (par exemple le principe de correspondance n'est pas un postulat fondamental).

- L'exploitation des propriétés de symétrie permet l'introduction la plus générale des propriétés physiques fondamentales : impulsion, moment angulaire... comme générateurs infinitésimaux de ces symétries, sans faire appel au principe de correspondance et à un analogue classique.

Les aspects pédagogiques ont fait l'objet d'une attention particulière. La progression des chapitres a été soigneusement étudiée, les chapitres 2 à 5 utilisant uniquement des espaces de dimension finie; c'est seulement une fois les bases acquises que l'on passe au cas général à partir du chapitre 6 . Un effort a été fait sur le vocabulaire, afin d'éviter certaines expressions historiquement datées et qui peuvent être un obstacle à la compréhension de la mécanique quantique : suivant la modernisation du vocabulaire préconisée par Lévy-Leblond [1999], "propriété physique" est utilisé au lieu d" "observable", "inégalité de Heisenberg" au lieu de "principe d'incertitude", des expressions comme "complémentarité" ou "dualité onde-corpuscule" ont été evitées, etc.

Les chapitres clés du livre, c'est-à-dire ceux qui divergent de la façon la plus évidente de l'exposé traditionnel, sont les chapitres 3, 4, 5 et 7. Le chapitre 3 introduit l'espace des états sur l'exemple de la polarisation des photons et montre comment passer d'une amplitude ondulatoire à une amplitude de probabilité. Le spin $1 / 2$ permet ensuite d'initier le lecteur à un problème sans analogue classique et à ses propriétés essentielles : algèbre des matrices de Pauli, matrices de rotation, précession de Larmor. Dans le chapitre 4, la distinction entre le cadre conceptuel général de la mécanique quantique et la modélisation d'un problème concret est soigneusement expliquée. Le chapitre 5 met en pratique la mécanique quantique sur des applications simples et physiquement importantes, dans le cas de systèmes dont le nombre de niveaux est fini. Il introduit l'interaction d'un système à deux niveaux atomique ou moléculaire avec un champ électromagnétique oscillant et développe des applications importantes : RMN, émission et absorption de photons, lasers, horloge atomiques... Le chapitre 7 a comme objectif l'étude des symétries à partir du théorème de Wigner, qui est généralement ignoré des manuels malgré son importance cruciale. La symétrie de rotation permet de définir le moment angulaire comme générateur infinitésimal et de démontrer immédiatement les relations de commutation de $\vec{J}$ en soulignant leur origine géométrique.

Le chapitre 11 sur l'intrication et la non-localité quantiques et les chapitres du deuxième tome pourront servir d'introduction à des sujets importants, et dont beaucoup sont apparus récemment : états intriqués, décohérence, information quantique, états du champ électromagnétique, intégrale de chemin, manipulation d'atomes par laser, condensats de Bose-Einstein, équations pilotes pour les systèmes ouverts et enfin mécanique quantique relativiste (équation de Dirac). Cette nouvelle édition, contrairement à la première qui 
était avant tout un livre d'enseignement, couvre une trop grande variété de domaines pour qu'elle puisse encore correspondre à un cours d'une année. Le guide de lecture ci-dessous devrait permettre au lecteur de s'orienter dans le livre pour un cours d'introduction à la mécanique quantique.

\section{Guide de lecture}

Seules les sections 1.3 à 1.5 du chapitre 1 sont indispensables pour la suite, et le chapitre 2 peut être omis par le lecteur qui possède le niveau L2 en algèbre. Les chapitres 3 et 5 constituent, à mon avis, le cour d'une introduction à la mécanique quantique : les principes de base sont introduits sur les exemples de la polarisation du photon et du spin $1 / 2$, et le rôle de la symétrie de rotation est mis en valeur dans le cas du spin 1/2. Des applications simples de ces principes de base sont exposées dans le chapitre 5 : résolution de l'équation de Schrödinger indépendante du temps pour des problèmes de chimie quantique élémentaire, oscillations de Rabi, le tout illustré par la RMN, l'émission et l'absorption de photons par un atome à deux niveaux, le laser et les horloges atomiques. Le chapitre 4 (Postulats de la mécanique quantique) est un peu plus abstrait : le lecteur pourra se contenter d'une première prise de contact, et y revenir après avoir acquis un peu plus de familiarité avec la mécanique quantique. Dans le chapitre 6, le lecteur peut se limiter à la section 6.3, qui donne les recettes "mathématiques" pour la suite. Les chapitres 7 à 10 sont classiques dans tout cours de mécanique quantique niveau L3. Toutefois la section 7.4 (invariance galiléenne) peut être omise sans dommage pour la suite et la section 8.4 (bandes d'énergie) peut trouver sa place dans un cours de physique du solide. Il en est de même pour la section 10.3 (niveaux de Landau) qui peut aussi faire partie d'un cours de physique du solide. Dans une première lecture du chapitre 11, on peut se limiter à l'introduction de l'opérateur statistique qui sera indispensable pour la suite. Il serait toutefois dommage de ne pas consacrer un peu de temps à la présentation élémentaire des inégalités de Bell (section 11.2). Le lecteur peut aborder les autres sections de ce chapitre, en particulier celle sur l'information quantique, en fonction de ses intérêts. Il en est de même pour les chapitres du tome 2 qui sont indépendants en première approximation; le lecteur familier du tome 1 peut les aborder dans un ordre indifférent. Le chapitre 12 (Méthodes semi-classiques) est entièrement optionnel, et ses quatre grandes parties : intégrale de chemin, approximation BKW, distribution de Wigner et phases géométriques sont largement indépendantes. Dans une première lecture, il est possible de se limiter aux sections 14.1 et $14.2 \mathrm{du}$ chapitre 14 (Particules identiques). Enfin les sections 15.1 et 15.2 sont une introduction standard à la physique atomique, les autres sections de ce chapitre étant optionnelles.

Remerciements. J'ai bénéficié des critiques et suggestions de Pascal Baldi, Jean-Pierre Farges, Yves Gabellini, Thierry Grandou, Jacques Joffrin, Jean-Marc Lévy-Leblond, Christian Miniatura et tout particulièrement de Michel Brune, Jean Dalibard, Franck Laloë, Fabrice Mortessagne, Jean-Pierre 
Romagnan, François Rocca et Sébastien Tanzilli qui ont lu de larges extraits du manuscrit. Je remercie également David Wilkowski qui a inspiré le texte de plusieurs exercices du chapitre 15. Je suis bien entendu entièrement responsable du texte final. L'aide de Karim Bernardet et de Fabrice Mortessagne a été décisive dans la réalisation des figures, et je tiens à remercier Christian Taggiasco pour sa compétence et sa disponibilité dans l'installation et la maintenance de l'ensemble des logiciels nécessaires. Enfin ce livre n'aurait pas vu le jour sans les encouragements et le soutien sans faille de Michèle Leduc, et je suis très reconnaissant à Claude Cohen-Tannoudji et Franck Laloë qui ont bien voulu le préfacer.

N.B. Cet ouvrage utilise le point décimal. 